\title{
Second pain event related potentials to argon laser stimuli: recording and quantification
}

\author{
L Arendt-Nielsen
}

\begin{abstract}
A non-invasive technique for quantification of argon laser induced burning second pain (C-fibre) is suggested. Using frequency analysis event related responses to burning pain can be detected in the EEG interval 1-2 seconds after laser stimulation. When the laser stimulus induced a burning pain perception, the power from $0.5-2.5 \mathrm{~Hz}$ of the EEG interval 1-2 seconds after stimulation differed significantly from the power calculated from the same time interval when no burning pain was perceived.
\end{abstract}

Non-invasive quantitative techniques for functional assessment of the unmyelinated nociceptive $\mathrm{C}$-fibre afferents do not exist. Techniques to stimulate the unmyelinated nociceptive Cfibres have been described but measurement and quantification of pain related responses are difficult. Attempts have been made to quantify C-fibre pain (the burning second pain) by electrical evoke responses recorded from the scalp). ${ }^{1}$

Strong thermal or electrical cutaneous stimuli can elicit two pain perceptions; a first pricking pain and a second more diffuse burning pain. The first pricking and the second burning pain perceptions are separated in time, due to different afferent conduction velocities for the corresponding A $\delta$-fibres (mean 19.2 $\mathrm{m} / \mathrm{s}^{2}$ and C-fibres (mean $0.86 \mathrm{~m} / \mathrm{s}^{3}$ ) The burning pain can be enhanced by blocking the A $\delta$-fibres or by increasing the stimulus rate. ${ }^{4}$

High energy lasers have been used for experimental pain stimulation, because they can induce pain without touching the skin. From model simulations it has been predicted that an infrared $\mathrm{CO}_{2}$ laser pulse $(10 \mathrm{~W}, 50 \mathrm{~ms}$ duration, $4 \mathrm{~mm}$ beam diameter) can increase the cutaneous temperature from $28^{\circ} \mathrm{C}$ to $50^{\circ} \mathrm{C}$ at a depth of $100 \mu \mathrm{m}$ below skin surface. ${ }^{5}$ This $\mathrm{CO}_{2}$ laser pulse intensity does not always provoke burning pain in addition to the pricking pain, although the cutaneous temperature at the receptor might have exceeded the firing threshold $\left(43-45^{\circ} \mathrm{C}\right)$ for the C-fibre innervated nociceptors. ${ }^{3,6}$ In contradiction Stowell ${ }^{7}$ concluded that $\mathrm{CO}_{2}$ laser stimulation was by no means as aversive as transcutaneous electrical stimulation.

Argon laser pulses with higher intensities than the pricking pain threshold are required to elicit the burning after-sensation. ${ }^{8}$ This indicates that the $\mathrm{C}$-fibres need a very fast rise-time of the intracutaneous temperature to elicit bursts of neural activity sufficient to evoke a well defined burning pain perception. The distinct $A \delta$-fibre mediated pain has been evaluated quantitatively by event related responses, ${ }^{9-18}$ but quantification of the C-fibre related laser induced responses has mainly been studied during pressure block of peripheral nerves. The latency of these C-fibre responses ranges from $900-1500 \mathrm{~ms}^{17}$

If $\mathrm{C}$-fibre related responses are to be used more widely, new and more adequate methods are needed. ${ }^{4}$ In this study strong argon laser stimulation was combined with a technique to analyse the frequency spectra of short EEG segments for activity related to or evoked by burning pain perception.

\section{Methods}

Volunteers

All volunteers gave their written consent. The study involved 12 males (mean age 23 years, range 20-26 years) and seven females (mean age 24 years, range 19-26 years). None had neurological or psychological disorders or were receiving medication. Each volunteer participated in two sessions. In the first session $\mathbf{9 0}$ argon laser stimuli were applied to the dorsum of the hand (C7-dermatome) and in the second session 90 stimuli were applied to the lateral part of the foot (S1-dermatome). The 90 stimuli were randomly distributed between three pain ratings, with 30 in each group. The procedures used for argon laser stimulation, EEG recording, and artifact rejection are described in the previous paper. ${ }^{16}$

\section{Subjective classification of laser stimuli}

The volunteers were initially trained to rate the intensity of the laser induced pain. The pain was classified as rating 1: distinct pinprick (moderate pain); rating 2: intense pinprick (strong pain), and rating 3: very intense pinprick followed by a burning after-sensation (severe pain). Fifteen seconds after stimulation the pain was rated, and the single responses were automatically stored in three databases according to the pain perceived. ${ }^{16}$ The mean laser intensities, related to each pain rating, were initially determined for each volunteer. These laser intensities were used for subsequent stimulation.

\section{Reaction time measurement}

A counter ( $1 / 1000 \mathrm{~s}$ resolution) was triggered at the onset of the stimulus. The counter was stopped by pressing a small thumb switch. The reaction times to the first pricking pain and to 
the second burning after-sensation were determined individually. Pain stimuli related to severe pain (rating 3 ) were used. The volunteers were trained intensively to react to the burning pain, and initially most found this difficult. Reaction times were calculated as the mean of 20 determinations. The intervals between stimuli were random with a mean of $20 \mathrm{~s}$ (range 10 to $30 \mathrm{~s}$ ). The reaction times to pricking pain and burning pain were determined before the EEG recording session.

Time domain analysis

Investigations were carried out to discover if the argon laser stimulation technique could evoke time locked responses related to the burning pain perception, and thus result in averaged responses in the time interval (1-2 s) as reported by Nauta and Bijl. ${ }^{1}$ The single responses were averaged according to the perceptual pain ratings: moderate, strong, or severe pain.

\section{Frequency domain analysis}

The spectral components of EEG activity can be calculated by a Fast Fourier Transform (FFT) algorithm or by an autoregressive model. For many purposes a FFT spectrum may be too noisy. Averaging of FFT spectra has been used to increase the signal to noise ratio. The alternative is to estimate the spectra by an autoregressive (AR) model, and the information in this predicted spectrum depends on the order of the model. ${ }^{19}$ AR spectra were calculated from one second EEG segments and no window function was used.

The EEG signal was divided into eight one second segments. The first segment contained EEG activity before the stimulus, the second segment contained the first pain related response, ${ }^{16}$ and the third segment was assumed to contain the second pain related response. In addition five subsequent segments were investigated. The AR frequency spectra of these EEG segments were calculated and averaged. The power in each of the eight segments was calculated in the interval from 0.5 to $12 \mathrm{~Hz}$ and the peak frequency was determined in the frequency interval from $0 \cdot 5-8 \mathrm{~Hz}$.

\section{Statistics}

Signed rank sum test (Wilcoxon's) was used for statistical analysis. Statistical significance was defined at the $5 \%$ level.

\section{Results}

Reaction time to pricking and burning pain At the start of the experiments the reaction times to pricking pain and burning pain were determined. The reaction time to the first pricking pain was $410+/-32 \mathrm{~ms}(+/-\mathrm{SD})$ for hand stimulation and $460+/-45 \mathrm{~ms}$ for foot stimulation. The reaction times to burning pain were $1330+/-290 \mathrm{~ms}$ for hand stimulation and $1630+/-390 \mathrm{~ms}$ for foot stimulation. The volunteers were instructed to react as soon as the burning pain started, because they found it easier to react to the onset of the burning pain than to the time of maximal burning pain. The burning pain increased rapidly to a maximal intensity and faded slowly away over 1000 to $2000 \mathrm{~ms}$. The mean difference in reaction times between hand and foot stimulation was $50 \mathrm{~ms}$ for pricking pain and $300 \mathrm{~ms}$ for burning pain.

\section{Time domain analysis}

To get an impression of the single responses to laser stimulation, pseudo three-dimensional plots were constructed. The EEG activity in the first second after stimulation was related to pricking pain and was found to be large and consistent (fig 1) for all pain ratings. In general no consistent later burning pain related responses were found. This indicates that if there was

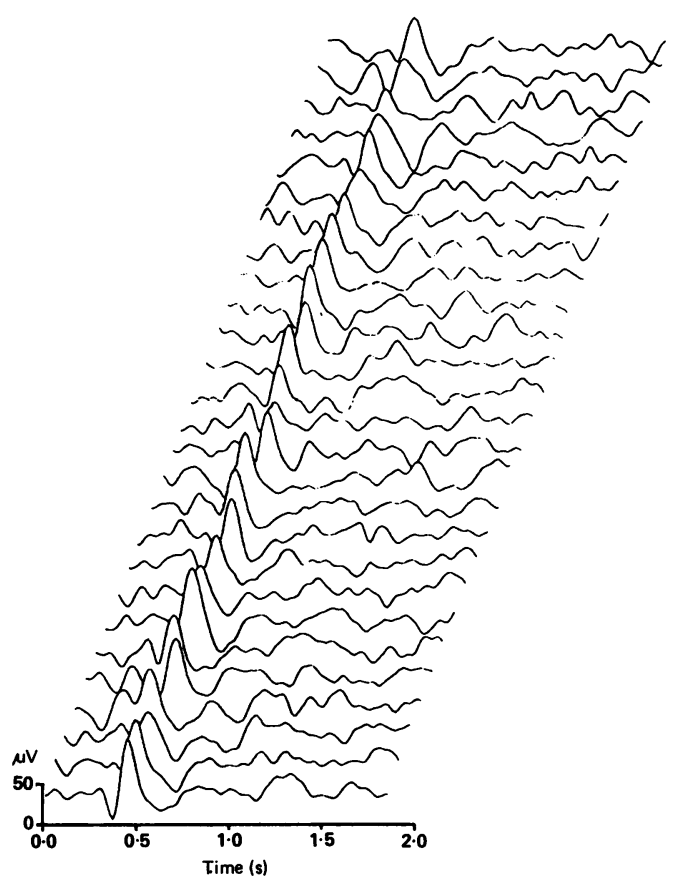

Figure 1 Pseudo three dimensional plot of 27 vertex recorded single responses to strong argon laser stimulation applied to the dorsum of the hand. The single responses were related to very strong pain followed by burning pain. In the first second after stimulation well defined first pain related responses were found whereas no reliable second pain related activity could be detected later after the stimulus. The EEG was filtered from $0.5 \mathrm{~Hz}$ to $12 \mathrm{~Hz}$ by second order filters.

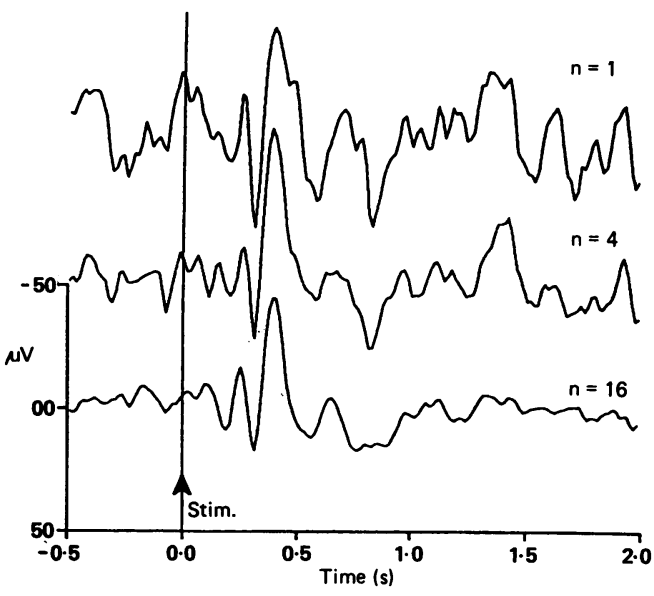

Figure 2 In some cases later $C$-fibre potentials could be detected 1-2 $s$ after stimulation in single trials or when averaging a few (for example, four). When averaging for example, 16 trials, the $C$-fibre response diminshed. The potentials shown were recorded from the vertex, and the strong laser pulses were applied to the dorsum of the hand. 
Figure 3 Superimposed traces of five seconds EEG following argon laser stimulation on the hand (C7-dermatome) and the foot (S1-dermatome).

Each trace was recorded from the vertex and 10 single potentials were averaged. The stimuli were rated as very intense pricking pain followed by burning pain (rating 3 ) and as intense pricking pain without any burning after-sensation (rating 2). The deflection between 0 $1 \mathrm{~s}$ is related to first pricking pain and the deflection between 1-2 s for pain rating 3 is possibly related to the burning $c-$ fibre mediated pain.
Figure 4 To find a sufficient order of the autoregressive model spectra for 1 s $E E G$ segments were calculated for different model orders $(P=5,10,15$, and 20$)$ The information in the spectra estimated by a 20 order model was not much different from the information obtained by a 15 order model. The argon laser stimulus (Stim.) was rated as very intense pain followed by burning pain. The power in first second after stimulation was related to pricking pain and the power in the interval from 1-2 s was related to burning pain. The displayed averaged response was recorded from vertex and evoked from the dorsum of the hand.

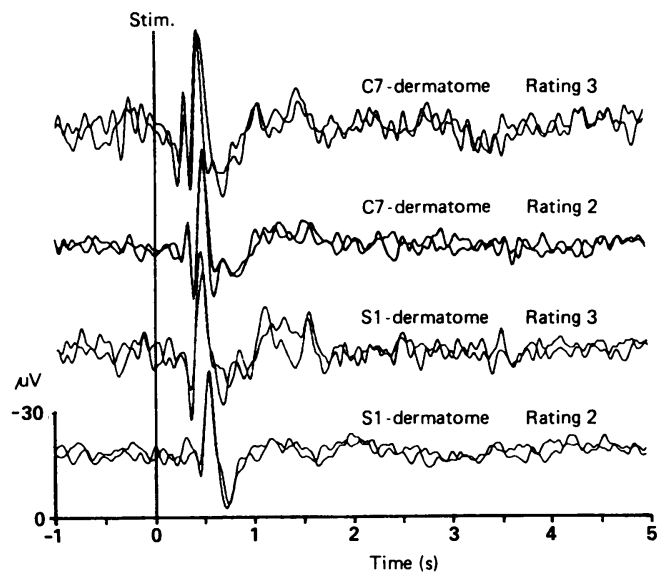

any activity related to the perception of burning pain, the signal would be of low amplitude and possibly of varying latency.

However, in three volunteers detectable responses $1-2 \mathrm{~s}$ after stimulation could be identified in the single trials and remained detectable when three to six trials were averaged. When additional responses were added, the response gradually disappeared (fig 2 ). This indicates that latency variation might be one of the reasons for the difficulty in detecting reliable averaged burning pain related responses.

In five other volunteers responses from both hand and foot stimulation could still be detected after averaging 10 trials. Two subsequent recordings were superimposed to study how reproducible the averaged responses were. The burning pain related activity remained stable (fig 3).

\section{FREQUENCY DOMAIN ANALYSIS}

The critical parameter for AR spectral estimation is the order of the model. The spectra should be used for feature extraction and it was therefore important that the spectra contained all the information needed. Estimated spectra for EEG segments before and after laser stimulation were systematically studied by
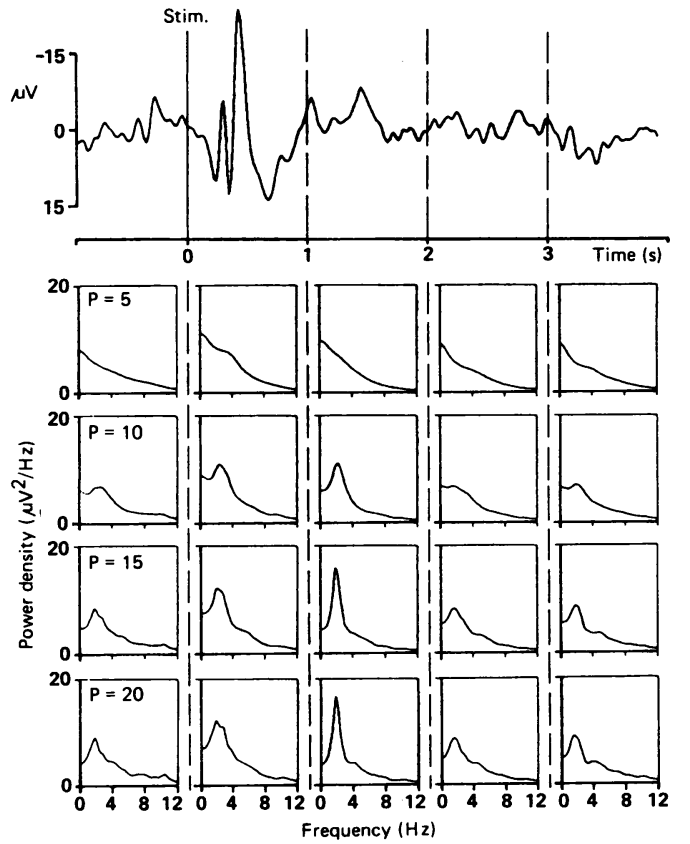

varying the model order from five to 20 . Theoretically a sufficient model order can be calculated using the Akaike Information Criteria. ${ }^{20}$ Using this criterion an order of 10 was found sufficient, but this method normally underestimates the order. ${ }^{21}$ An order of 15 was chosen (fig 4).

To avoid the effect of latency variations of the single trials the frequency spectra of the individual trials were calculated. The spectra contain an amplitude and a phase information, where the phase contains information concerning, for example, latencies. The amplitude spectrum contains information concerning, for example, slow waves. The EEG signal was divided into short one second epochs and each epoch was analysed. Using an AR model it is possible to analyse short time sequences and still estimate the low frequencies, and this model is ideal for predicting peaks in the spectrum. If the spectra of individual trials are averaged peaks can be detected if they in mean are larger than the background activity. It should, however, be emphasised that spectral averaging does not reduce the variance of the noise (background EEG) as seen when averaging in the time domain.

The spectral components of the first and second pain EEG segments differed from the pre-stimulus EEG activity. The total power of the first pain related response was normally
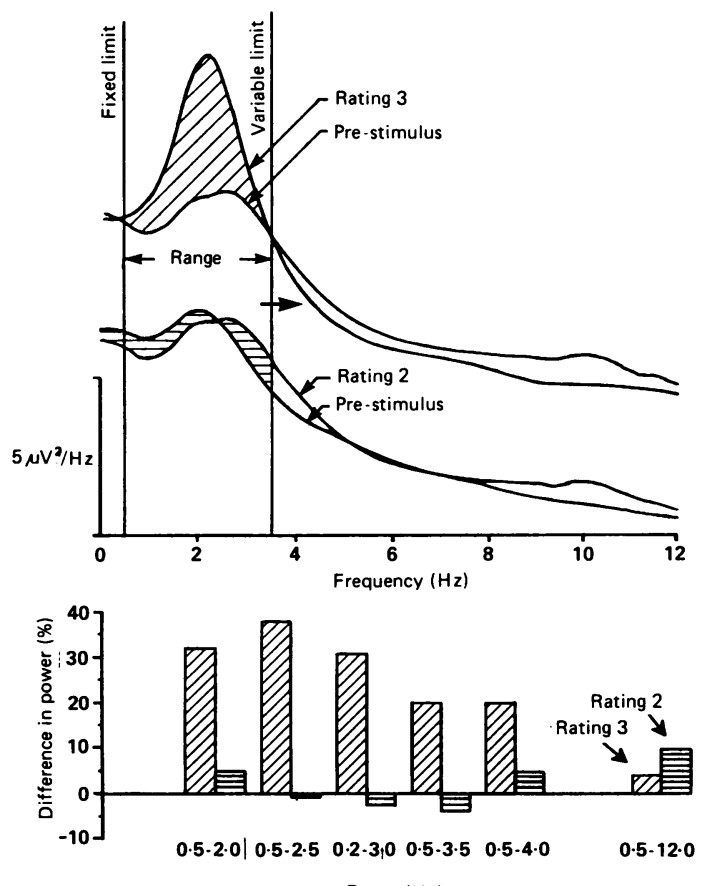

Range $(\mathrm{Hz})$

Figure 5 The power density spectra $\left(\mu V^{2} / H z\right)$ of $E E G$ segments recorded before the laser stimulation (prestimulus), and 1-2 s after stimulation (C-fibre segment). The spectra are compared for pain rating 3 (top) and 2. $A$ spectrum for pain rating 1 is not shown because it is similar to that for pain rating 2. The differences in power between the EEG spectra before stimulation and the $C$-fibre spectra are symbolised by hatched areas for pain rating $3(||)$ and $2(=)$. The power (bottom) was calculated in the range between $0.5 \mathrm{~Hz}$ (fixed limit) and $2 \cdot 0,2 \cdot 5,3 \cdot 0,3 \cdot 5,4 \cdot 0$ and $12 \cdot 0 \mathrm{~Hz}$ (variable limit). The maximal percentage difference in power between prestimulus EEG and $C$-fibre responses was found in the range from $0.5-2.5 \mathrm{~Hz}$. Pain rating 3 was very intense pricking pain followed by burning pain and for pain rating 2 no burning pain was reported. 
larger than the other segments. For the second pain segment the differences were not that clear, and attempts were therefore made to optimise the frequency interval in which the largest difference to background EEG could be obtained.

The difference in power between the EEG activity before the stimulus and the C-fibre segment was calculated for different frequency intervals. A maximal difference of $36 \%$ was found for the 0.5 to $2.5 \mathrm{~Hz}$ range (fig 5). For moderate and strong pain stimuli no systematic differences were found between the segments.

Power in the c-fibre segment (1-2 s after stimulation)

The power $(0.5-2.5 \mathrm{~Hz})$ of the averaged single spectrum was calculated for the EEG segment before the stimulus, for the first pain related segment, for the second pain related segment, and for the five following EEG segments. When the results from the 19 volunteers were analysed five observations could be made:

1) For moderate and strong pain stimuli (no burning pain) the power in the C-fibre segment was not statistically different from the EEG segment before stimulus, and from the five EEG segments 2-7 s after stimulation.

2) The power in the $C$-fibre segment for severe pain (burning pain) stimuli differed significantly from the same segment for moderate ( $\mathrm{p}<0.03)$ and strong ( $<<0.03)$ pain stimuli (no burning pain).

3) For severe pain stimuli (burning pain) the power in the C-fibre segment differed significantly $(p<0.02)$ from the EEG segment before the stimulus.

4) The power in the C-fibre segment differed significantly $(p<0.04-p<0.02)$ from five post-stimulus (2-7 s) segments for severe pain stimuli (burning pain).

5) The total power from $0.5-2.5 \mathrm{~Hz}$ of the first pain related response $\left(138 \mu \mathrm{V}^{2}\right)$ was in mean $30 \%$ higher than the power of the C-fibre response $\left(110 \mu \mathrm{V}^{2}\right)$ for severe pain stimuli (burning pain).

The power in the C-fibre response recorded from vertex was approximately $30 \%$ higher than the ipsi- and contralateral recordings. This does not, however, automatically imply that the signal-to-noise ratio is highest for the vertex recording. The difference between power in the EEG segment before stimulus and the C-fibre EEG segment was highest for the vertex recording, which indicates that the best signal-to-noise ratio was obtained from the vertex recording.

\section{Peak frequency of the $C$-fibre segment}

(-2 s after stimulation)

The mean peak frequency below $8 \mathrm{~Hz}$ of the averaged spectra was determined for the 19 volunteers. Five observations were made:

1) The mean peak frequency of the first pain response $(2.42+/-0.31 \mathrm{~Hz})$ was significantly $(\mathrm{p}<0.03)$ higher than that of the C-fibre segment $(2 \cdot 21+/-0.27 \mathrm{~Hz})$ for severe pain stimulation (burning pain).

2) For moderate and strong pain stimuli (no burning pain) no significant differences occurred between the peak frequency of the EEG segment before the stimulus and the Cfibre segment.

3) The peak frequency of the C-fibre segment for severe pain stimuli (burning pain) differed significantly from the same segment for moderate $(\mathrm{p}<0.04)$ and strong $(\mathrm{p}<0.03)$ pain stimuli (no burning pain).

4) For severe pain stimulation (burning pain) the peak frequency of the C-fibre segment $(2.21 \mathrm{~Hz})$ differed significantly $(p<0.005)$ from the EEG segment before the stimulus $(2 \cdot 45 \mathrm{~Hz})$.

5) The peak frequency of the C-fibre segment $(2.21 \mathrm{~Hz})$ differed significantly $(\mathrm{p}<0.04-\mathrm{p}<$ $0.005)$ from the post-stimulus segments $(2-7 \mathrm{~s})$ for severe pain stimulation (burning pain).

By combining the peak frequency and the power parameter, it was possible to detect event related C-fibre responses from $80 \%$ of the volunteers. The remaining volunteers all reported that the pricking pain was very predominant, compared with the burning pain perception. The power and the peak frequency of the C-fibre segment both differed significantly from other segments when burning pain was perceived. The activity detected may therefore reflect the central processing of and attention to C-fibre mediated pain.

\section{Discussion}

Reaction time to burning pain

During pressure block on the radial nerve Bromm and Treede ${ }^{17}$ found a reaction time of $1350 \mathrm{~ms}$ to $\mathrm{CO}_{2}$ laser evoked burning pain. Before block they found a mean reaction time of $480 \mathrm{~ms}$ to pricking pain. In this study the reaction times to pricking and burning pain were $470+/-32 \mathrm{~ms})(+\mid-\mathrm{SD})$ and $1330+\mid$ $-470 \mathrm{~ms}$, respectively.

In a recent study Arendt-Nielsen and Bjerring ${ }^{22}$ used $\mathrm{CO}_{2}$ and visible argon laser stimulation to elicit pricking pain, and found that the reaction times were highly dependent on skin conditions and the intensity of the laser stimulus. This should be taken into consideration when reaction times and hence the latency of the responses, evoked from different dermatomes, are compared. The stimulus and skin conditions were therefore carefully controlled in this study. The differences in reaction times to hand and foot stimulation were $50 \mathrm{~ms}$ for the pricking pain and $300 \mathrm{~ms}$ for the burning pain, giving a factor of six in difference between conduction times. This is slightly lower than what should be expected on the basis of the peripheral conduction velocities where the range of $A \delta$-fibre peripheral conduction velocities is 4 to $30 \mathrm{~m} / \mathrm{s}$ (mean $19.2 \mathrm{~ms}^{2}$ ), and for C-fibre they are 0.5 to $1.4 \mathrm{~ms}$ (mean $0.86 \mathrm{~ms}^{3}$ ), giving a mean factor of 22. Part of this discrepancy can be explained by the involvement of central pathways when reaction times are determined.

Perception of laser induced $C$-fibre pain Initially the volunteers were trained to rate the intensity of the burning pain, but as they found 
this task very difficult the scoring was excluded from the experiment. It has therefore not been possible to correlate the quantitative parameters (peak frequency and power) to the intensity of the burning pain perceived. The averaged power spectra did not take the latency variations into consideration, which is a disadvantage because latency variations may, somehow, correlate with the quality of the pain perceived or the stimulus intensity.

In general, the volunteers reported that the burning pain sensation was strong for severe pain stimulation (rating 3). Argon laser pulses have previously been used for pain stimulation due to the optimal interaction between argon laser light and the skin. ${ }^{8}$ The light from the optical fibre is assumed to be absorbed directly by the free nerve endings and by haemoglobin and melanin. ${ }^{23}$ The direct transmission of the light to the nerve endings permits very high intracutaneous temperature rise-times without burning the superficial skin layers as often seen for $\mathrm{CO}_{2}$ laser stimulation. These high risetimes might be ideal for $\mathrm{C}$-fibre activation. Comparing the quality of argon and $\mathrm{CO}_{2}$ laser induced pain, we experienced that the argon lasers evoked a more distinct and unpleasant pain perception compared to $\mathrm{CO}_{2}$ laser stimulation. Stowell ${ }^{7}$ has reported that $\mathrm{CO}_{2}$ laser stimuli are perceived as less painful than electrical stimuli.

Time domain analysis of $C$-fibre responses The activity related to $C$-fibre pain was assumed to occur between one and two seconds after stimulation. The amplitude of C-fibre related activity was, under normal conditions, comparable to the amplitude of the general EEG activity. During A $\delta$-fibre block Bromm and Treede $^{17}$ found $\mathrm{C}$-fibre responses with peak-to-peak amplitudes to be similar to single $\mathrm{A} \delta$-fibre responses $(30-50 \mu \mathrm{V})$.

The amplitudes of the $A \delta$-fibre first pain related single responses are large and related to the intensity of the stimulus and hence the pricking pain perceived. ${ }^{1624}$ These large deflections were not generally seen for the C-fibre responses on the 3D-plots. However, occasionally the first responses in a series showed a detectable activity one to two seconds after stimulation. When the averaging procedure continued, the C-fibre related activity gradually disappeared. This may be due to habituation because the first pain related response in a series is always the largest. ${ }^{16}$ Another explanation may be that the pricking pain perception modulates the attention to the burning pain component. The evoked responses are reduced if the volunteer is aware of the stimulus timing, ${ }^{25}$ or if the time between stimuli is reduced. ${ }^{426}{ }^{27}$ Central inhibition of Cfibre activity by the $A \delta$-fibres might be another possibility. Katz et $a^{28}$ showed an interaction between unspecific large and small diameter fibres on the electrically evoked responses in anaesthetised cats.

Latency jittering may be the major obstacle for detecting averaged C-fibre responses. ${ }^{17}$ Attempts have been made ${ }^{17}$ to improve the representation of the $\mathrm{C}$-fibre response by latency corrected averaging (Woody filtering). ${ }^{29}$ In this technique the single responses are aligned before averaging. Due to the low signal-to-noise ratio for the $\mathrm{C}$-fibre responses we did not use this technique. It is highly uncertain if reliable results can be obtained, because responses with similar amplitude and shape as averaged C-fibre responses can occur when the EEG intervals before the stimuli are used as input to the filter. ${ }^{16}$

Conventional averaging of C-fibre responses has been investigated for one subject by Nauta and Bijl. ${ }^{1}$ They used strong electrical stimulation and found an early complex at $300 \mathrm{~ms}$, and a late broad ( $800 \mathrm{~ms}$ duration) complex with a peak latency of $1550 \mathrm{~ms}$. In contradiction averaging of $\mathrm{CO}_{2}$ laser induced burning pain responses did not result in any late complex..$^{30}$ By using argon laser stimulation, it was found in five cases out of 19 that averaged C-fibre responses could be detected for both hand and foot stimulation. The duration of the responses ranged from 200 to $500 \mathrm{~ms}$ with peak latency from $900-1600 \mathrm{~ms}$.

C-fibre responses could be detected from three recording sites; $\mathrm{C} 3, \mathrm{Cz}$, and $\mathrm{C} 4$ but the best signal-to-noise ratio was obtained from vertex. The scalp topography of the $\mathrm{C}$-fibre response recorded during pressure block, has indicated that the maximal amplitude is obtained over vertex ${ }^{30}$ similar to that found for first pain related responses. ${ }^{1631}$

Frequency domain analysis of $C$-fibre responses The mean peak frequency of the first pain related response was significantly higher than for the C-fibre EEG segment, indicating a longer duration of the $\mathrm{C}$-fibre related response. This could be explained by the larger, temporal dispersion of the C-fibre volley, caused by the low conduction velocity $(0.5-2.5 \mathrm{~m} / \mathrm{s})$. A more optimal C-fibre interval for frequency analysis could possibly be found, but this needs further investigation.

In conclusion, both peak frequency and power of the EEG in the one to two seconds interval after strong argon laser stimulation correlated with the perception of second pain (burning pain). The vertex recorded response showed the best signal-to-noise ratio, which is similar to the finding for first pain related response. We believe that the detected responses reflect a sequence of central events rather than a direct projection of the neural transmission of C-fibre activity. The potential might be similar to the vertex potential (event related potential) but just delayed. The vertex potential can be elicited by abrupt changes in perception, independent of the stimulus modality.

A non-invasive technique to evaluate a response related to the thin afferent pain sensitive $\mathrm{C}$-fibre has been suggested. The technique can be combined with quantification of the $A \delta$ fibre mediated pricking pain perception. In clinical neurophysiology many attempts have been made to assess the functioning of the pain sensitive $A \delta$ - and C-fibres. The technique presented may enable future quantitative studies of the function of these afferents. 
I am grateful to Professor Annelise Rosenfalck for her helpful suggestions. This work was supported by the Danish Cancer Society (Grant no. 87-006), the Danish Medicalm Research Council (12-7547), and the Danish Technical Research Counci $(16-4061)$.

1 Nauta J, Bijl GK. Second pain and related cortical potentials. In: Nodar RH, Barber C, eds. Evoked potentials II. London: Butterworth, 1984:379-81.

2 Adriaensen H, Gybels J, Handwerker HO, Van Hess J. Response properties of thin myelinated $(\mathrm{A}-\delta)$ fibres in human skin nerves. $J$ Neurophysiol 1983;49:111-22.

3 Van Hess J, Gybels J. C nociceptor activity in human nerve during painful and non painful skin stimulation. $J$ Neurol Neurosurg Psychiatry 1981;44:600-7.

4 Harkins SW, Price DD, Katz MA. Are cerebral evoked potentials reliable indices of first or second pain. In: Bonica JJ, Lindblom U, Ainsley I, eds. Advances in pain research and therapy, Vol 5. New York: Raven Press, 1983:185-91

5 Haimi-Cohen $R$, Cohen A, Carmon A. A model for the temperature distribution in skin noxiously stimulated by a brief pulse of $\mathrm{CO}_{2}$ laser radiation. J Neurosci Meth 1983;8:127-37.

6 Hallin RG, Torebjork HE, Wiesenfeld Z. Nociceptors and warm receptors innervated by $\mathrm{C}$-fibres in human skin. weurol Neurosurg Psychiatry 1981;44:313-9.

7 Stowell $\mathrm{H}$. Event related brain potentials and human pain: first objective overview. Int J Psychophysiology 1984, 1:137-51.

8 Arendt-Nielsen L, Bjerring P. Sensory and pain threshold characteristics to laser stimuli. J Neurol Neurosutg Psychiatry 1988;51:35-42.

9 Carmon A, Mor J, Goldberg J. Evoked cerebral responses to noxious thermal stimuli in humans. Exp Brain Res 1976;25:103-7.

10 Carmon A, Dotan Y, Sarne Y. Correlation of subjective pain experience with cerebral evoked responses to noxious thermal stimulations. Exp Brain Res 1978;33:445-53.

11 Carmon A. Considerations of the cerebral responses to painful stimulation: Stimulus transduction versus percep-

12 Carmon A, Friedman Y, Coger R, Kenton B. Single trial analysis of evoked potentials to noxious thermal stimulation in man. Pain 1980;8:21-32.

13 Coger RW, Kenton B, Pinsky JJ, Crue BL, Carmon A, Friedman Y. Somatosensory evoked potentials and noxious stimulation in patients with intractable noncancer pain syndromes. Psychiatry Res 1980;2:279-94.

14 Kenton B, Coger R, Crue B, Pinsky J, Friedman Y, Carmon A. Peripheral fibre correlates to noxious thermal stimulation in humans. Neurosci Lett 1980;17:301-6.

15 Bromm B, Treede R-D. Nerve fibre discharges, cerebral potentials and sensations induced by $\mathrm{CO}_{2}$ laser stimulation. Human Neurobiol 1984;3:33-40.

16 Arendt-Nielsen L. First pain event related potentials to argon laser stimuli-recording and quantification. $J$ to argon laser stimuli-recording and quan

17 Bromm B, Treede RD. Human cerebral potentials evoked by $\mathrm{CO}_{2}$ laser stimuli causing pain. Exp Brain Res 1987; $\mathrm{CO}_{2}$ laser

18 Arendt-Nielsen L, Bjerring P. Selective averaging of argon laser induced pre-pain and pain related cortical responses. $J$ Neurosci Meth 1988;24:117-23.

19 Makhoul J. Linear prediction: A tutorial review. Proc IEEE 1975;63:561-80.

20 Akaike H. Recent development of statistical methods for spectrum estimation. In: Yamaguchi N, Fujisawa K, eds. Recent advantages in EEG and EMG data processing. Amsterdam: Elsevier, 1984:63-78.

21 Kay SM, Marple SL. Spectrum analysis-A modern perspective. Proc IEEE 1981;71:1380-419.

22 Arendt-Nielsen L, Bjerring P. Reaction time to painless and painful laser stimulation. Eur J Appl Physiol 1988;58: 266-73.

23 Parrish J, Anderson R. Considerations of selectivity in laser therapy. In: Arndt KA, Noe JM, Rosen S, eds. Cutaneous laser therapy: Principles and methods. New York: Wiley and Sons, 1983:41-52.

24 Bjerring P, Arendt-Nielsen L. Argon laser induced single cortical responses: a new method to quantify pre-pain perceptions. J Neurol Neurosurg Psychiatry 1988;51:43-9.

25 Schafer EWP, Amochaev A, Russell MJ. Knowledge of stimulus timing attenuates human evoked cortical potentials. Electroenceph Clin Neurophysiol 1981;52:9-17.

26 Angle RW, Quick WM, Boylls CC, Weinrich M, Rodnitzky RL. Decrement of somatosensory evoked potentials during repetitive stimulation. Electroenceph Clin Neurophysiol 1985;60:335-42.

27 Jacobson RC, Chapman CR, Gerlach R. Stimulus intensity and interstimulus interval effects on pain-related cerebral potentials. Electroenceph Clin Neurophysiol 1985;62: potentials.

28 Katz S, Martin HF, Blackburn JG. The effects of interaction between large and small diameter fibre systems on the somatosensory evoked potentials. Electroenceph Clin Neurophysiol 1978;45:45-52.

29 Woody CD. Characterisation of an adaptive filter for analysis of variable latency neuroelectric signals. Med Biol Eng 1967;5:539-53.

30 Treede RD, Bromm B. Reliability and validity of ultra-late cerebral potentials in response to C-fibre activation in man. In: Dubner R, Gebhart $R$, Bond MR, ed. Proc Vth World Congress on Pain. Amsterdam: Elsevier, 1988: 567-73.

31 Treede RD, Kief S, Hölzer T, Bromm B. Late somatosensory evoked potentials in response to cutaneous heat stimulation. Electroenceph Clin Neurophysiol 1988; 70:429-41. 